\title{
PSEUDO MV-ALGEBRAS ARE INTERVALS IN $\ell$-GROUPS
}

\author{
ANATOLIJ DVUREČENSKIJ
}

(Received 7 March 2000; revised 23 May 2001)

\author{
Communicated by B. Davey
}

\begin{abstract}
We show that any pseudo MV-algebra is isomorphic with an interval $\Gamma(G, u)$, where $G$ is an $\ell$-group not necessarily Abelian with a strong unit $u$. In addition, we prove that the category of unital $\ell$-groups is categorically equivalent with the category of pseudo MV-algebras. Since pseudo MV-algebras are a non-commutative generalization of MV-algebras, our assertions generalize a famous result of Mundici for a representation of $\mathrm{MV}$-algebras by Abelian unital $\ell$-groups. Our methods are completely different from those of Mundici. In addition, we show that any Archimedean pseudo MV-algebra is an MV-algebra.
\end{abstract}

2000 Mathematics subject classification: primary 03B50, $03 \mathrm{G} 12$.

Keywords and phrases: pseudo MV-algebra, MV-algebra, unital $\ell$-group, strong unit, categorical equivalence, Archimedean pseudo MV-algebras.

\section{Introduction}

Recently, Georgescu and Iorgulescu [11] introduced pseudo MV-algebras which are a non-commutative generalization of $\mathrm{MV}$-algebras introduced in 1958 by Chang [4] and which are a generalization of a two-valued reasoning. A non-commutative generalization of reasoning can be found for example in psychological processes: In clinical medicine on behalf of transplantation of human organs, an experiment was performed in which the same two questions have been posed to two groups of interviewed people: (1) Do you agree to donate your organs for medical transplantation after your death? (2) Do you agree to accept organs of a donor if you need them? If the order of questions was changed in the second group, the number of positive answers here was much higher than in the first group.

According to [11], we say that a pseudo $M V$-algebra is an algebra $\left(M ; \oplus,^{-}, \sim, 0,1\right)$ of type $(2,1,1,0,0)$ such that the following axioms hold for all $x, y, z \in M$ with an

(C) 2002 Australian Mathematical Society $1446-7887 / 2000 \$ A 2.00+0.00$ 
additional binary operation $\odot$ defined via $y \odot x=\left(x^{-} \oplus y^{-}\right)^{\sim}$
(Al) $x \oplus(y \oplus z)=(x \oplus y) \oplus z$
(A2) $x \oplus 0=0 \oplus x=x$;
(A3) $x \oplus 1=1 \oplus x=1$;
(A4) $1^{\sim}=0 ; 1^{-}=0$;
(A5) $\left(x^{-} \oplus y^{-}\right)^{\sim}=\left(x^{\sim} \oplus y^{\sim}\right)^{-}$;
(A6) $x \oplus x^{\sim} \odot y=y \oplus y^{\sim} \odot x=x \odot y^{-} \oplus y=y \odot x^{-} \oplus x$;
(A7) $x \odot\left(x^{-} \oplus y\right)=\left(x \oplus y^{\sim}\right) \odot y$;
(A8) $\left(x^{-}\right)^{\sim}=x$.

For example, if $G$ is an $\ell$-group (not necessarily Abelian) and if $u$ is a positive element in it, then $(\Gamma(G, u) ; \oplus, \sim, \sim, u)$, where

$$
\begin{array}{cc}
\Gamma(G, u):=\{x \in G: 0 \leq x \leq u\}, \\
x \oplus y:=(x+y) \wedge u, & x^{\sim}:=-x+u, \\
x \odot y:=(x-u+y) \vee 0, & x^{-}:=u-x,
\end{array}
$$

is a pseudo MV-algebra.

On the other hand, Rachunek [15] introduced non-commutative MV-algebras, which in fact are equivalent with pseudo $\mathrm{MV}$-algebras. The equivalence is given by the fact that if $\left(M ; \oplus, \odot,^{-}, \sim, 0,1\right)$ is a pseudo MV-algebra, then $\left(M ; \oplus ; \odot^{\prime}, \neg, \sim, 0,1\right)$, where $x \odot^{\prime} y:=y \odot x$, and ${ }^{-}=^{-}$, is a non-commutative MV-algebra. Conversely, if $(M ; \oplus, \odot, \neg, \sim, 0,1)$ is a non-commutative MV-algebra, then $\left(M ; \oplus, \odot^{\prime},-, \sim, 0,1\right)$, where $x \odot^{\prime} y=y \odot x$ and ${ }^{-}={ }^{-}$, is a pseudo MV-algebra.

It is clear that classical MV-algebras are pseudo MV-algebras, and for them Mundici [14] proved a crucial fact that any MV-algebra $M$ is isomorphic with some $\Gamma(G, u)$, where $G$ is an Abelian $\ell$-group with a strong unit $u$ (a positive element $u \in G$ is said to be a strong unit of $G$ if given $g \in G$, there exists an integer $n \geq 1$ such that $-n u \leq g \leq n u$ ). In addition, he proved the categorical equivalence of the category of MV-algebras with the category of unital $\ell$-groups.

The aim of the present paper is to show that also any pseudo MV-algebra is isomorphic with some unit interval $\Gamma(G, u)$ given with a strong unit $u$ of an $\ell$-group $G$ by (1.1)-(1.2), and that the category of unital $\ell$-groups is categorically equivalent with the category of pseudo MV-algebras. In addition, we show that any Archimedean pseudo MV-algebra is a usual MV-algebra. Our method is completely different from that used by Mundici, and it depends on Bosbach's notion of a semiclan [3]. Moreover, we give positive answers to problems formulated in [9] and [7].

\section{Partial addition in pseudo MV-algebras}

Throughout the paper, we assume that $\left(M ; \oplus,^{-}, \sim, 0,1\right)$ is a pseudo MV-algebra. 
If we define $x \leq y$ if and only if $x^{-} \oplus y=1$, then $\leq$ is a partial order such that $M$ is a distributive lattice with $x \vee y=x \oplus x^{\sim} \odot y$, and $0 \leq x \leq 1$ (we recall that $\odot$ has a higher priority than $\oplus$ ). For basic properties of pseudo MV-algebras see [11].

Define a partial binary operation + on $M$ via: $x+y$ is defined if and only if $x \leq y^{-}$, and in this case $x+y:=x \oplus y$.

It is clear that $x+y$ is defined if and only if $x \leq y^{-}$if and only if $y \leq x^{\sim}$. The following has been proved in [7] or in [9].

PROPOSITION 2.1. The following properties hold in any pseudo $M V$-algebra $M$.

(i) $x+0=x=0+x$ for any $x \in M$.

(ii) $x+x^{2}=1=x^{-}+x$ for any $x \in M$.

(iii) If $x^{-}+y=1$, then $y=x$.

(iv) If $x+y=1$, then $y=x^{\sim}$ and $x=y^{-}$.

(v) Let $x+y$ and $(x+y)+z$ be defined in $M$. Then $y+z$ and $x+(y+z)$ are defined in $M$, and $(x+y)+z=x+(y+z)$. Similarly, if $y+z$ and $x+(y+z)$ are defined in $M$, so are $x+y$ and $(x+y)+z$.

(vi) If $x+b=y$, then $b=x^{\sim} \odot y$.

(vii) If $x+y_{1}=x+y_{2}$, then $y_{1}=y_{2}$.

(viii) If $x_{1}+y=x_{2}+y$, then $x_{1}=x_{2}$.

(ix) If $y=a+x$, then $a=y \odot x^{-}$.

(x) If $x \wedge y=0$, then $x+y$ and $y+x$ are defined in $M$, and $x+y=x \vee y=y+x$.

PROPOSITION 2.2. (i) If $a+b_{1}$ and $a+b_{2}$ exist in $M$, then $a+\left(b_{1} \vee b_{2}\right)$ is defined in $M$, and $a+\left(b_{1} \vee b_{2}\right)=\left(a+b_{1}\right) \vee\left(a+b_{2}\right)$.

(ii) If $b_{1}+a$ and $b_{2}+a$ exist in $M$, then $\left(b_{1} \vee b_{2}\right)+a$ is defined in $M$, and $\left(b_{1} \vee b_{2}\right)+a=\left(b_{1}+a\right) \vee\left(b_{2}+a\right)$.

(iii) If $a+b_{1}$ and $a+b_{2}$ exist in $M$, then $a+\left(b_{1} \wedge b_{2}\right)$ is defined in $M$, and $a+\left(b_{1} \wedge b_{2}\right)=\left(a+b_{1}\right) \wedge\left(a+b_{2}\right)$.

(iv) If $b_{1}+a$ and $b_{2}+a$ exist in $M$, then $\left(b_{1} \wedge b_{2}\right)+a$ is defined in $M$, and $\left(b_{1} \wedge b_{2}\right)+a=\left(b_{1}+a\right) \wedge\left(b_{2}+a\right)$.

PROPOSITION 2.3. For all $x$, $y$ of a pseudo $M V$-algebra $M$, we have

(a) $x \odot(x \wedge y)^{-}=x \odot y^{-}$

(b) $x \odot(x \wedge y)^{-}+y=x \odot y^{-}+y=x \vee y$;

(c) $(x \wedge y)^{\sim} \odot y=x^{\sim} \odot y, x \vee y=x+(x \wedge y)^{\sim} \odot y$;

(d) $(x \vee y) \odot y^{-}=x \odot(x \wedge y)^{-}=x \odot y^{-}$;

(e) $y^{\sim} \odot(x \vee y)=y^{\sim} \odot x=(x \wedge y)^{\sim} \odot x$.

PROPOSITION 2.4. In any pseudo $M V$-algebra $M$ we have the following cancellation laws. 
(i) If $a+b_{1} \leq a+b_{2}$, then $b_{1} \leq b_{2}$.

(ii) If $a_{1}+b \leq a_{2}+b$, then $a_{1} \leq a_{2}$.

(iii) If $a+b$ is defined in $M$, and if $a_{1} \leq a$ and $b_{1} \leq b$, then $a_{1}+b_{1}$ is defined in $M$, and $a_{1}+b_{1} \leq a+b$.

The notion of a semiclan introduced by Bosbach [3] will be crucial for our reasoning.

We say that $(C ; \wedge,+)$ is a semiclan if it is a $\wedge$-semilattice and a partial groupoid with respect to + such that the following axioms are satisfied:

(C1) If $a \leq b$, then there exist $x, y \in C$ such that $b=a+x$ and $b=y+a$.

(C2) If $a+x, a+y \in C, a+x=a+y$, then $x=y$, and if $x+a, y+a \in C$, $x+a=y+a$, then $x=y$.

(C3) If $a+x, a+y \in C$, then $(a+x) \wedge(a+y)=a+(x \wedge y)$, and if $x+a, y+a \in C$, then $(x+a) \wedge(y+a)=(x \wedge y)+a$.

(C4) $a+b,(a+b)+c \in C$ if and only if $b+c, a+(b+c) \in C$, and in this case we have $(a+b)+c=a+(b+c)$.

(C5) If $(a \wedge b)+c=c$ and $a \vee b$ exists, then $a+b=a \vee b=b+a$.

It is clear that if $G$ is an $\ell$-group, then $\left(G^{+} ; \wedge,+\right)$ is a semiclan, and by Bosbach [3, page 321], for any semiclan $(C ; \wedge,+)$ there exists an $\ell$-group $G$ with the positive cone $G^{+}$such that $C$ can be embedded into some semiclan $\left(G^{+} ; \wedge,+\right)$ preserving + , $\wedge$, and all existing $\vee$ in $C$.

PROPOSITION 2.5. Let $\left(M ; \oplus,-,^{\sim}, 0,1\right)$ be a pseudo $M V$-algebra and let + be defined as above. Then $(M ; \wedge,+)$ is a semiclan, and, in addition, there exist an $\ell$-group $G_{0}$ with the positive cone $G_{0}^{+}$and an injective mapping $f: M \rightarrow G_{0}^{+}$such that $f$ preserves,$+ \wedge$, and $\vee$.

Proof. Using Proposition 2.1-Proposition 2.4 , we see that $(M ; \wedge,+)$ is a semiclan. Using the crucial result of Bosbach [3, page 321] mentioned above, we have the assertion in question.

\section{Embedding of pseudo MV-algebras onto $\Gamma(G, u)$}

In the present section, we show that any pseudo MV-algebra $M$ is isomorphic with some $\Gamma(G, u)$.

Throughout this section, let $f$ and $G_{0}$ from Proposition 2.5 be fixed.

Denote by $X_{0}:=f(M)$ and $X:=\left\{\sum_{i=1}^{n} f\left(a_{i}\right): a_{1}, \ldots, a_{n} \in M, n \geq 1\right\}$. Then $X_{0}$ is a lattice which is a sublattice of $G_{0}^{+}$. We claim below that $X$ is also a sublattice of $G_{0}^{+}$; for $x, y \in X$, the expression $x \wedge y \in X$ means that $x \wedge y$ taken in the $\ell$-group $G_{0}$ is an element of $X$. 
PROPOSITION 3.1. If $f\left(a_{1}\right)+\cdots+f\left(a_{n}\right)=f(b)$, where $a_{1}, \ldots, a_{n}, b \in M$, then $a_{1}+\cdots+a_{n}$ exists in $M$, and $b=a_{1}+\cdots+a_{n}$.

PROOF. We will argue by the mathematical induction on $n$. If $n=1$, the statement is evident. Assume now that the statement holds for each $i \leq n$, and let $f\left(a_{1}\right)+$ $\cdots+f\left(a_{n}\right)+f\left(a_{n+1}\right)=f(b)$. Because $b=b \odot a_{n+1}^{-}+a_{n+1}$, we have $f(b)=$ $f\left(b \odot a_{n+1}^{-}\right)+f\left(a_{n+1}\right)$. Using the cancellation property holding in any $\ell$-group, we have $f\left(a_{1}\right)+\cdots+f\left(a_{n}\right)=f\left(b \odot a_{n+1}^{-}\right)$, and consequently, $b=b \odot a_{n+1}^{-}+a_{n+1}=$ $a_{1}+\cdots+a_{n}+a_{n+1}$.

PROPOSITION 3.2. If $f\left(a_{1}\right)+\cdots+f\left(a_{n}\right) \leq f(b)$, where $a_{1}, \ldots, a_{n}, b \in M$, then $a_{1}+\cdots+a_{n}$ exists in $M$, and $a_{1}+\cdots+a_{n} \leq b$.

PROOF. We will follow the mathematical induction on $n$. If $n=1$, the statement is evident. Assume now that the statement holds for each $i \leq n$, and let $f\left(a_{1}\right)+\cdots+$ $f\left(a_{n+1}\right) \leq f(b)$. Then $f(b)=f\left(b \odot a_{n+1}^{-}\right)+f\left(a_{n+1}\right)$, consequently, $f\left(a_{1}\right)+\cdots+$ $f\left(a_{n}\right) \leq f\left(b \odot a_{n+1}^{-}\right)$, which yields $a_{1}+\cdots+a_{n} \leq b \odot a_{n+1}^{-}$. Hence, there exists $a_{0} \in M$ such that $a_{0}+\left(a_{1}+\cdots+a_{n}\right)=b \odot a_{n+1}^{-}$. In view of $f(b)=f\left(a_{0}\right)+f\left(a_{1}+\cdots+\right.$ $\left.a_{n}\right)+f\left(a_{n+1}\right)$ and due to Proposition 3.1, we have $b=a_{0}+\left(a_{1}+\cdots+a_{n}\right)+a_{n+1}$.

PROPOSITION 3.3. Given $a_{1}, \ldots, a_{n} \in M, b_{1}, \ldots, b_{m} \in M$, there exist elements $c_{1}, \ldots, c_{r} \in M$ and $d_{1}, \ldots, d_{s} \in M$ such that

$$
\sum_{i=1}^{n} f\left(a_{i}\right)+\sum_{j=1}^{m} f\left(b_{j}\right)=\sum_{k=1}^{r} f\left(c_{k}\right)+\sum_{i=1}^{n} f\left(a_{i}\right),
$$

and

$$
\sum_{i=1}^{n} f\left(a_{i}\right)+\sum_{j=1}^{m} f\left(b_{j}\right)=\sum_{j=1}^{m} f\left(b_{j}\right)+\sum_{t=1}^{s} f\left(d_{t}\right) .
$$

Proof. (a) Let $a, b \in M$. Then

$$
a=a \odot\left(a \odot b^{-}\right)^{-}+a \odot b^{-}, \quad b=b \odot a^{-}+a \wedge b .
$$

By [11, Proposition 1.24], $a \odot b^{-} \wedge b \odot a^{-}=0$, and $a \odot b^{-}+b \odot a^{-}=b \odot a^{-}+a \odot b^{-}$. Then

$$
\begin{aligned}
f(a)+f(b) & =f\left(a \odot\left(a \odot b^{-}\right)^{-}\right)+f\left(a \odot b^{-}\right)+f\left(b \odot a^{-}\right)+f(a \wedge b) \\
& =f\left(a \odot\left(a \odot b^{-}\right)^{-}\right)+f\left(b \odot a^{-}\right)+f\left(a \odot b^{-}\right)+f(a \wedge b) \\
& =f\left(a \odot\left(a \odot b^{-}\right)^{-}\right)+f\left(b \odot a^{-}\right)+f\left(a \odot(a \wedge b)^{-}\right)+f(a \wedge b) \\
& =f\left(a \odot\left(a \odot b^{-}\right)^{-}\right)+f\left(b \odot a^{-}\right)+f(a) .
\end{aligned}
$$


Similarly, we have $a=a \wedge b+b^{\sim} \odot a, b=a^{\sim} \odot b+\left(a^{\sim} \odot b\right)^{\sim} \odot b$.

By [11, Proposition 1.24] $b^{\sim} \odot a \wedge a^{\sim} \odot b=0$, so that $b^{\sim} \odot a$ and $a^{\sim} \odot b$ commute, and

$$
\begin{aligned}
f(a)+f(b) & =f(a \wedge b)+f\left(b^{\sim} \odot a\right)+f\left(a^{\sim} \odot b\right)+f\left(\left(a^{\sim} \odot b\right)^{\sim} \odot b\right) \\
& =f(a \wedge b)+f\left(a^{\sim} \odot b\right)+f\left(b^{\sim} \odot a\right)+f\left(\left(a^{\sim} \odot b\right)^{\sim} \odot b\right) \\
& =f(b)+f\left(b^{\sim} \odot a\right)+f\left(\left(a^{\sim} \odot b\right)^{\sim} \odot b\right) .
\end{aligned}
$$

(b) Using the mathematical induction, we have guaranteed the existence of $\left\{c_{u}\right\}$ in $M$ such that the following chain of equalities hold

$$
\begin{aligned}
f(a) & +f\left(b_{1}\right)+\cdots+f\left(b_{m+1}\right) \\
& =\left(f(a)+f\left(b_{1}\right)\right)+\left(f\left(b_{2}\right)+\cdots+f\left(b_{m+1}\right)\right) \\
& =\left(f\left(c_{1}\right)+\cdots+f\left(c_{u}\right)\right)+f(a)+\left(f\left(b_{2}\right)+\cdots+f\left(b_{m+1}\right)\right) \\
& =f\left(c_{1}\right)+\cdots+f\left(c_{u}\right)+\left(f\left(c_{u+1}\right)+\cdots+f\left(c_{k}\right)\right)+f(a) .
\end{aligned}
$$

(c) Similarly,

$$
\begin{aligned}
f\left(a_{1}\right) & +\cdots+f\left(a_{n+1}\right)+f(b) \\
& =\left(f\left(a_{1}\right)+\cdots+f\left(a_{n}\right)\right)+\left(f\left(a_{n+1}\right)+f(b)\right) \\
& =\left[\left(f\left(a_{1}\right)+\cdots+f\left(a_{n}\right)\right)+f(b)\right]+\left(f\left(d_{t}\right)+\cdots+f\left(d_{s}\right)\right) \\
& =f(b)+f\left(d_{1}\right)+\cdots+f\left(d_{t-1}\right)+f\left(d_{t}\right)+\cdots+f\left(d_{s}\right) .
\end{aligned}
$$

Combining (a)-(c), we have (3.1) and (3.2).

Proposition 3.4. Let $A, B, U \in X$. If $A \wedge B \in X, B-(A \wedge B) \in X$, and $U \wedge(B-(A \wedge B)) \in X$, then $(U+A) \wedge B \in X$

ProOF. Since $A, B, U$ are elements of $G_{0}^{+}$, we have

$$
\begin{aligned}
& U \wedge(B-(A \wedge B))+(A \wedge B) \\
& \quad=(U+A \wedge B) \wedge B=(U+A) \wedge(U+B) \wedge B=(U+A) \wedge B
\end{aligned}
$$

which proves the assertion in question.

PROPOSITION 3.5. For all $a_{1}, \ldots, a_{n}, b \in M,\left(f\left(a_{1}\right)+\cdots+f\left(a_{n}\right)\right) \wedge f(b) \in X$.

ProOF. The proof will follow the mathematical induction on $n$.

If $\boldsymbol{n}=1$, the statement is trivial. Suppose now that it holds for any $f\left(a_{1}\right)+$ $\cdots+f\left(a_{i}\right)$ with $1 \leq i \leq n$. Put $A=f\left(a_{2}\right)+\cdots+f\left(a_{n+1}\right), U=f\left(a_{1}\right)$, and $B=f(b)$. By induction, $A \wedge B \in X$. Since $A \wedge B \leq f(b)$, we conclude by 
Proposition 3.2 that there exists an element $v \in M$ such that $f(v)=A \wedge B \in X_{0}$. Moreover, $B-(A \wedge B)=f(b)-f(v)=f\left(b \odot v^{-}\right) \in X_{0} \subset X$. Therefore, $U \wedge(B-(A \wedge B)) \in X_{0}$. Using Proposition 3.4, $(U+A) \wedge B \in X$, that is, $\left(f\left(a_{1}\right)+\cdots+f\left(a_{n+1}\right)\right) \wedge f(b) \in X$.

We now present the following form of the Riesz decomposition property.

PROPOSITION 3.6. Let $f(c) \leq f\left(a_{1}\right)+\cdots+f\left(a_{n}\right)$, where $c, a_{1}, \ldots, a_{n} \in M$. Then there exists $c_{1}, \ldots, c_{n} \in M$ with $c_{i} \leq a_{i}, i=1, \ldots, n$, such that $f(c)=$ $f\left(c_{1}\right)+\cdots+f\left(c_{n}\right)$.

PROOF. Let us apply the induction on $n$. If $n=1$, then $c \leq a_{1}$. Suppose now that the assertion holds for any $i$ with $1 \leq i \leq n$. Thus let $f(c) \leq f\left(a_{1}\right)+\cdots+f\left(a_{n+1}\right)$. Define $v=c \odot(b \wedge c)^{-} \in M, b=a_{n+1}, A=f\left(a_{1}\right)+\cdots+f\left(a_{n}\right)$, and $A_{1}=$ $\left(f\left(a_{1}\right)+\cdots+f\left(a_{n}\right)\right) \wedge f(v)$. By Proposition 3.5, $A_{1} \in X$, and since $A_{1} \leq f(v)$, there exists $a_{0} \in M$ such that $A_{1}=f\left(a_{0}\right)$. It is clear that $a_{0} \leq v \leq c$. Put $c_{n+1}=a_{0}^{\tilde{C}} \odot c$. Then $c=a_{0}+a_{0}^{\tilde{c}} \odot c$. Since $f\left(a_{0}\right) \leq A=f\left(a_{1}\right)+\cdots+f\left(a_{n}\right)$, by the induction hypothesis there exist $c_{1}, \ldots, c_{n} \in M$ with $c_{i} \leq a_{i}, 1 \leq i \leq n$, and $f\left(c_{1}\right)+\cdots+f\left(c_{n}\right)=f\left(a_{0}\right) \leq f\left(a_{1}\right)+\cdots+f\left(a_{n}\right)$. Then

$$
\begin{aligned}
f\left(a_{0}\right)+f\left(c_{n+1}\right) & =f(c) \leq(A+f(b)) \wedge(f(b) \vee f(c)) \\
& =(A+f(b)) \wedge\left(f\left(c \odot b^{-}\right)+f(b)\right) \\
& =(A+f(b)) \wedge\left(f\left(c \odot(b \wedge c)^{-}\right)+f(b)\right) \\
& =\left(A \wedge f\left(c \odot(b \wedge c)^{-}\right)\right)+f(b)=f\left(a_{0}\right)+f(b),
\end{aligned}
$$

which by entails $f\left(c_{n+1}\right) \leq f(b)$, and $c_{n+1} \leq b=a_{n+1}$, and the proof is finished.

PROPOSITION 3.7. If $f(c) \leq f\left(a_{1}\right)+\cdots+f\left(a_{n}\right)$, where $a_{1}, \ldots, a_{n}, c \in M$, then $f\left(a_{1}\right)+\cdots+f\left(a_{n}\right)-f(c) \in X$ and $-f(c)+f\left(a_{1}\right)+\cdots+f\left(a_{n}\right) \in X$.

PROOF. Applying Proposition 3.6, we can find elements $c_{1}, \ldots, c_{n} \in M$ such that $c_{i} \leq a_{i}, i=1, \ldots, n$, and $f(c)=f\left(c_{1}\right)+\cdots+f\left(c_{n}\right)$. Then $f\left(c_{1}\right)+\cdots+f\left(c_{n-1}\right) \leq$ $f\left(a_{1}\right)+\cdots+f\left(a_{n-1}\right)+f\left(a_{n}\right)-f\left(c_{n}\right)=f\left(a_{1}\right)+\cdots+f\left(a_{n-1}\right)+f\left(a_{n} \odot c_{n}^{-}\right)$. Using Proposition 3.3, we can find elements $x_{1}, \ldots, x_{k_{1}} \in M$ such that

$$
\begin{aligned}
& \left(f\left(a_{1}\right)+\cdots+f\left(a_{n-1}\right)\right)+f\left(a_{n} \odot c_{n}^{-}\right) \\
& \quad=f\left(x_{1}\right)+\cdots+f\left(x_{k_{1}}\right)+f\left(a_{1}\right)+\cdots+f\left(a_{n-1}\right) \in X .
\end{aligned}
$$

Successively repeating this procedure, we can prove the assertion in question. In the same way we proceed for the second equality. 
PROPOSITION 3.8. $X$ is a lattice semigroup which is naturally ordered, that is, if $x, y \in X$ and $x \leq y$, then $-x+y \in X$ and $y-x \in X$.

PrOOF. It is clear that $X$ is a partially ordered semigroup, and by Proposition 3.7, $X$ is naturally ordered.

We show that $X$ is a lattice. By Proposition 3.5, $\left(f\left(a_{1}\right)+\cdots+f\left(a_{n}\right)\right) \wedge f(b) \in X$ for all $a_{1}, \ldots, a_{n}, b \in M$. We have to prove that $\left(f\left(a_{1}\right)+\cdots+f\left(a_{n}\right)\right) \wedge\left(f\left(b_{1}\right)+\right.$ $\left.\cdots+f\left(b_{k}\right)\right) \in X$. We will apply the complete induction on $k$.

If $k=1$, we apply Proposition 3.5. Suppose now that the assertion holds for any $j$ with $1 \leq j \leq k$. Put $B=f\left(a_{1}\right)+\cdots+f\left(a_{n}\right) \in X, A=f\left(b_{2}\right)+\cdots+f\left(b_{k+1}\right)$, $U=f\left(b_{1}\right)$. By the induction assumption, $A \wedge B \in X$, and by Proposition 3.7, $B-(A \wedge B) \in X$. By Proposition 3.5, $U \wedge(B-(A \wedge B)) \in X$, so that by Proposition 3.4, $\left(f\left(a_{1}\right)+\cdots+f\left(a_{n}\right)\right) \wedge\left(f\left(b_{1}\right)+\cdots+f\left(b_{k+1}\right)\right)=(U+A) \wedge B \in X$.

We are now ready to prove the main theorem of Section 3.

THEOREM 3.9. Let $\left(M ; \oplus,^{-}, \sim, 0,1\right)$ be a pseudo $M V$-algebra. Then there exists an $\ell$-group $G$ with strong unit $u$ such that $M$ and $\Gamma(G, u)$ are isomorphic pseudo MV-algebras.

PrOOF. Let $G_{0}$ and $f$ be defined in Proposition 2.5. In Proposition 3.8 we have proved that $X$ is an $\ell$-semigroup of $G_{0}^{+}$, so: (i) $X$ is left and right cancellative, (ii) $0=f(0)$ is the neutral element, (iii) if $x+y=0$ for $x, y \in X$ then $x=0=y$. By Proposition 3.3, $x+X=X+x$ for $x \in X$. Applying now the Birkhoff theorem, [10, Theorem II.4], we see that $X$ is a positive cone of an $\ell$-group $G$. By the construction of $X, u:=f(1)$ is a strong unit for $G$. In view of Proposition 3.2, $\Gamma(G, u)=f(M)$.

Let $a \in M$. Then $a^{-}+a=1=a+a^{\sim}$ hence $f\left(a^{-}\right)+f(a)=u=: f(1)=$ $f(a)+f\left(a^{\sim}\right)$ which gives $f\left(a^{-}\right)=f(a)^{-}$and $f\left(a^{\sim}\right)=f(a)^{\sim}$.

Let $a, b \in M$ be given. Then $a \oplus b=a+a^{\sim} \odot(a \oplus b)=a+a^{\sim} \wedge b$ so that

$$
f(a \oplus b)=f(a)+f\left(a^{\sim} \wedge b\right)=f(a)+f(a)^{\sim} \wedge f(b)=f(a) \oplus f(b),
$$

where $f(a) \oplus f(b)$ is now taken in $\Gamma(G, u)$. It is clear that $f(0)=0$.

REMARK 3.10. We note that if $M$ is a commutative pseudo $M V$-algebra, that is, $a \oplus b=b \oplus a, a, b \in M$, then ${ }^{-}=^{\sim}$, and $M$ is an MV-algebra, and in Theorem 3.9 we have obtained that $G$ is an Abelian $\ell$-group, which gives the most important part of Mundici's representation of MV-algebras by $\Gamma(G, u)$. Our proof is completely different from Mundici's one who used the notion of good sequences. Another proof of such a representation of $\mathrm{MV}$-algebras can be found in [8], where it was obtained in the frame of BCK-algebras using Wyler's [17] and Baer's [1] technique of words. 


\section{Archimedean pseudo MV-algebras}

We introduce Archimedean pseudo MV-algebras which will entail the commutativity of pseudo MV-algebras.

According to [7] and [9], we say that a pseudo MV-algebra $M$ is Archimedean if the existence of $n a:=a_{1}+\cdots+a_{n}$, where $a_{1}=\cdots=a_{n}=a$ for any integer $n \geq 1$, entails that $a=0$. It is known that if $M$ is a $\sigma$-complete lattice, then $M$ is Archimedean, [9, Proposition 6.4.14]. In [9], it was proved that an Archimedean pseudo MV-algebra $M$ is commutative if (i) it is atomic, [9, Theorem 6.4.20], in particular if $M$ is finite, or if (ii) $M$ is linear, [9, Theorem 6.4.23].

In the present section, we prove that any Archimedean pseudo MV-algebra is commutative.

PROPOSITION 4.1. Let $\left(M ; \oplus,^{-}, \sim, 0,1\right)$ be a pseudo $M V$-algebra. Let $f$ and $(G, u)$ be those from Theorem 3.9. Then $M$ is Archimedean if and only if $G$ is an Archimedean $\ell$-group.

ProOF. Let $G$ be Archimedean, then $\Gamma(G, u)$ is an Archimedean pseudo MValgebra, so $M$ is Archimedean.

Conversely, let $M$ be an Archimedean pseudo MV-algebra, then $\Gamma(G, u)$ is Archimedean.

To prove that $G$ is Archimedean, it is sufficient to verify that if, for any $n \geq 1$, $n g \leq v$ for $g \geq 0, g, v \in G$, then $g=0$. Indeed, the general case of $g \in G$ follows from the simple considerations: $n g^{+}=(n g)^{+}=(n g) \vee 0 \leq v \vee 0=v^{+}$which implies $g^{+}=0$ and, therefore, $g=g^{+}-g^{-}=-g^{-} \leq 0$.

Assume that $n g \leq v$ for $g, v \in G^{+}$and for each $n \geq 1$. Since $f(M)$ generates the positive cone $G^{+}$, there exist finitely many elements $y_{1}, \ldots, y_{k}$ in $M$ such that

$$
v=\sum_{i=1}^{k} f\left(y_{i}\right) .
$$

The proof will use the induction on $k$.

Case 1. Assume that $v=f(y)$ for some $y \in M$. Since $g \leq f(y)$, according to Theorem 3.9, there is a unique $x \in M$ such that $g=f(x)$. Since $n f(x) \leq f(y)$ for any integer $n \geq 1$ and $n f(x) \in G^{+}$, applying Proposition 3.2, we conclude that $n x$ is defined in $M$ for any integer $n \geq 1$. Hence $n f(x)=f(n x) \leq f(y)$ which entails $n x \leq y$ for any $n \geq 1$. The hypothesis gives $x=0$, and consequently $g=0$.

Case 2. Suppose we have proved the problem in question for any element $g \in G^{+}$ and for any element $v$ of the form (4.1), where the summation in (4.1) is over integers $\leq k$. Assume now $n g \leq v=\sum_{i=1}^{k} f\left(y_{i}\right)+f(y)$ for $n \geq 1$, where $y=y_{k+1} \in X$. 
Then $n g-f(y) \leq \sum_{i=1}^{k} f\left(y_{i}\right)$ for any integer $n \geq 1$. In lattice ordered groups we have $j(s \vee 0)=(s j) \vee(j-1) s \vee \cdots \vee s \vee 0$ for any integer $j \geq 1$ (see [2, page 293]). Therefore, for all $n, m \geq 1$, we have

$$
\begin{aligned}
m[(n g & -f(y)) \vee 0] \\
& =m(n g-f(y)) \vee(m-1)(n g-f(y)) \vee \cdots \vee(n g-f(y)) \vee 0 \\
& \leq[m n g-f(y)] \vee[(m-1) n g-f(y)] \vee \cdots \vee(n g-f(y)) \vee 0 \\
& \leq \sum_{i=1}^{k} f\left(y_{i}\right) \vee \sum_{i=1}^{k} f\left(y_{i}\right) \vee \cdots \vee \sum_{i=1}^{k} f\left(y_{i}\right) \vee 0=\sum_{i=1}^{k} f\left(y_{i}\right) .
\end{aligned}
$$

Since (ng $-f(y)) \vee 0 \in G^{+}$applying the induction hypothesis, we conclude that $(n g-f(y)) \vee 0=0$ for any integer $n \geq 1$. Therefore, $n g \leq f(y)$ for all $n \geq 1$. We can now apply Case 1 to conclude $g=0$.

THEOREM 4.2. Any Archimedean pseudo MV-algebra is commutative, that is, an MV-algebra.

Proof. Suppose that $M$ is isomorphic with $\Gamma(G, u)$, where $G$ is an $\ell$-group with strong unit $u$. According to Proposition 4.1, $G$ is an Archimedean $\ell$-group, and the Archimedeanicity of $G$ is according to [10, Corollary V.20] or [2, page 317] a sufficient condition to be $G$ a commutative $\ell$-group. So that $\Gamma(G, u)$ and $M$ are commutative pseudo MV-algebras.

It is well known that any poset $M$ has the MacNeille completion by cuts, say $\hat{M}$. $\hat{M}$ is a complete lattice in which $M$ can be embedded preserving all existing joins and meets in $M$. By Schmidt [16], the MacNeille completion $\hat{M}$ is (up to isomorphism) a lattice in which $M$ can be supremum-densely and infimum-densely embedded (that is, for any element $x \in \hat{M}$ there exist two subsets $U, Q$ of $M$ such that $x=\bigvee_{\hat{M}} \phi(U)=$ $\bigwedge_{\hat{M}} \phi(Q)$ (where $\phi$ is the embedding of $M$ into $\hat{M}$ preserving all existing joins and meets in $M)$.

THEOREM 4.3. A pseudo MV-algebra $M$ has the MacNeille completion as a pseudo $M V$-algebra if and only if $M$ is Archimedean.

Proof. Suppose that the MacNeille completion $\hat{M}$ of a pseudo MV-algebra $M$ is a pseudo MV-algebra. Therefore as it was mentioned above, $\hat{M}$ is Archimedean, and consequently, $M$ is Archimedean.

Conversely, let $M$ be an Archimedean pseudo MV-algebra. By Theorem 4.2, $M$ is commutative and an MV-algebra. Since an MV-algebra $M$ is Archimedean if and only if $M$ is semisimple, [9], by Höhle [12, Theorem 6.3], $\hat{M}$ is a complete MV-algebra. 
Using the Scrimger 2-group, [6, page 20], we present an example of a countable pseudo MV-algebra which is not commutative and hence not Archimedean.

EXAMPLE 4.4. Let $G=(\mathbb{Z} \times \mathbb{Z} \times \mathbb{Z} ;+,(0,0,0), \leq)$ be the Scrimger 2-group, that is,

$$
\left(k_{1}, m_{1}, n_{1}\right)+\left(k_{2}, m_{2}, n_{2}\right):= \begin{cases}\left(k_{1}+m_{2}, m_{1}+k_{2}, n_{1}+n_{2}\right) & \text { if } n_{2} \text { is odd } \\ \left(k_{1}+k_{2}, m_{1}+m_{2}, n_{1}+n_{2}\right) & \text { if } n_{2} \text { is even. }\end{cases}
$$

Then $0=(0,0,0)$ is the neutral element, and

$$
-(k, m, n)= \begin{cases}(-m,-k,-n) & \text { if } n_{2} \text { is odd; } \\ (-k,-m,-n) & \text { if } n_{2} \text { is even, }\end{cases}
$$

and $G$ is a non-Abelian $\ell$-group with the positive cone

$$
G^{+}=\mathbb{Z} \times \mathbb{Z} \times \mathbb{Z}_{>0}^{+} \cup \mathbb{Z}^{+} \times \mathbb{Z}^{+} \times\{0\},
$$

or equivalently, $\left(k_{1}, m_{1}, n_{1}\right) \leq\left(k_{2}, m_{2}, n_{2}\right)$ if and only if (i) $n_{1}<n_{2}$, or (ii) $n_{1}=n_{2}$, $k_{1} \leq k_{2}, m_{1} \leq m_{2}$. Then

$$
\left(k_{1}, m_{1}, n_{1}\right) \vee\left(k_{2}, m_{2}, n_{2}\right):= \begin{cases}\left(k_{1}, k_{2}, n_{1}\right) & \text { if } n_{1}>n_{2} ; \\ \left(k_{1} \vee k_{2}, m_{1} \vee m_{2}, n_{1} \vee n_{2}\right) & \text { if } n_{1}=n_{2} ; \\ \left(k_{2}, m_{2}, n_{2}\right) & \text { if } n_{1}<n_{2},\end{cases}
$$

and $u=(1,1,1)$ is a strong unit for $G$. Consequently, the corresponding pseudo MV-algebra is not Archimedean (for example, $n(1,1,0)=(n, n, 0) \in \Gamma(G, u)$ for any $n \geq 1$ ) and has the form

$$
\Gamma(G, u)=\mathbb{Z}^{+} \times \mathbb{Z}^{+} \times\{0\} \cup \mathbb{Z}_{\leq 1} \times \mathbb{Z}_{\leq 1} \times\{1\},
$$

with

$$
\begin{array}{ll}
(k, m, 0)^{-}=(1-k, 1-m, 1), & (k, m, 0)^{\sim}=(1-m, 1-k, 1), \\
(k, m, 1)^{-}=(1-m, 1-k, 0), & (k, m, 1)^{\sim}=(1-k, 1-m, 0),
\end{array}
$$

and

$$
\begin{aligned}
& \left(k_{1}, m_{1}, 0\right) \oplus\left(k_{2}, m_{2}, 0\right)=\left(k_{1}+k_{2}, m_{1}+m_{2}, 0\right), \\
& \left(k_{1}, m_{1}, 0\right) \oplus\left(k_{2}, m_{2}, 1\right)=\left(\left(m_{1}+k_{2}\right) \wedge 1,\left(m_{2}+k_{1}\right) \wedge 1,1\right), \\
& \left(k_{1}, m_{1}, 1\right) \oplus\left(k_{2}, m_{2}, 0\right)=\left(\left(k_{1}+k_{2}\right) \wedge 1,\left(m_{1}+m_{2}\right) \wedge 1,1\right), \\
& \left(k_{1}, m_{1}, 1\right) \oplus\left(k_{2}, m_{2}, 1\right)=(1,1,1) .
\end{aligned}
$$




\section{Universal group}

We say that a partially ordered group $(G ;+, \leq, 0)$ with a mapping $f: M \rightarrow G^{+}$ is a universal group for a pseudo $\mathrm{MV}$-algebra $\left(M ; \odot,^{-}, \sim, 0,1\right)$ if

(i) the positive cone $G^{+}$is generating for $G$, that is, $G=G^{+}-G^{+}$;

(ii) $f(M)$ generates $G^{+}$as a semigroup;

(iii) $f(x+y)=f(x)+f(y)$ whenever $x+y$ exists in $M, x, y \in M$;

(iv) for any group $K$ and any + preserving mapping $h: M \rightarrow K$, there is a group homomorphism $\phi: G \rightarrow K$ such that $h=\phi \circ f$.

The universal group, if it exists, is unique up to isomorphism, and $\phi$ from (iv) is a unique group homomorphism with that property. We denote the universal group for $M$ by $\mathscr{G}=(G, f)$.

In what follows, we show that if $f$ and $G$ are those from Theorem 3.9 , then $(G, f)$ is a universal group for $M$.

Throughout the rest of this section, let $f$ be any injective mapping from $M$ into the positive cone $G^{+}$of an $\ell$-group $G$ preserving + and $\wedge$, and $\vee$. In other words, $f$ is an embedding of the semiclan $(M ; \wedge,+)$ into the semiclan $\left(G^{+} ; \wedge,+\right)$, and for it all results of the beginning of the present section are valid, too. Moreover, $f(M)=\Gamma\left(G^{\prime}, u\right)$ for $u=f(1)$, where $G^{\prime}$ is an $\ell$-subgroup of $G$.

We start with variations of the Riesz decomposition property.

PROPOSITION 5.1. Let $f\left(a_{1}\right)+f\left(a_{2}\right)=f\left(b_{1}\right)+f\left(b_{2}\right), a_{1}, a_{2}, b_{1}, b_{2} \in M$. Then there exist four elements $c_{11}, c_{12}, c_{21}, c_{22} \in M$ such that

$$
\begin{array}{ll}
a_{1}=c_{11}+c_{12}, & b_{1}=c_{11}+c_{21}, \\
a_{2}=c_{21}+c_{22}, & b_{2}=c_{12}+c_{22} .
\end{array}
$$

Moreover, we may assume that $c_{12} \wedge c_{21}=0$, and under this condition the $c_{i j}$ are determined uniquely.

PROOF. We define $c_{11}:=a_{1} \wedge b_{1}, c_{12}:=b_{1}^{\sim} \odot a_{1}=\left(b_{1} \wedge a_{1}\right)^{\sim} \odot a_{1}=\tilde{c_{11}} \odot a_{1}$, $c_{22}:=a_{2} \wedge b_{2}$, and $c_{21}:=a_{2} \odot b_{2}^{-}=a_{2} \odot\left(a_{2} \wedge b_{2}\right)^{-}=a_{2} \odot c_{22}^{-}$.

Then $a_{1}=c_{11}+c_{12}$ and $a_{2}=c_{21}+c_{22}$. We now show that $a_{2} \odot b_{2}^{-}=a_{1}^{-} \odot b_{1}$. Put $y=f\left(a_{1}\right)+f\left(a_{2}\right)=f\left(b_{1}\right)+f\left(b_{2}\right)$. Then $y=f\left(b_{1}\right)-f\left(b_{1}\right)+y=y-f\left(b_{2}\right)+$ $f\left(b_{2}\right)=f\left(a_{1}\right)-f\left(a_{1}\right)+y=y-f\left(a_{2}\right)+f\left(a_{2}\right)$. By the cancellation property holding in $\ell$-groups, we have $f\left(b_{2}\right)=-f\left(b_{1}\right)+y, f\left(b_{1}\right)=y-f\left(b_{2}\right), f\left(a_{1}\right)=y-f\left(a_{2}\right)$, and $f\left(a_{2}\right)=-f\left(a_{1}\right)+y$.

We claim $f\left(c_{21}\right)=f\left(a_{1}^{\sim} \odot b_{1}\right)$. Indeed, the equality $a \vee b=a+a^{\sim} \odot b=$ $b \odot a^{-}+a$ implies $f(a) \vee f(b)=f(a)+f\left(a^{\sim} \odot b\right)=f\left(b \odot a^{-}\right)+f(a)$. Therefore, $f\left(b \odot a^{-}\right)=0 \vee(f(b)-f(a)), f\left(a^{\sim} \odot b\right)=0 \vee(-f(a)+f(b))$. Then 
$f\left(c_{21}\right)=f\left(a_{2} \odot b_{2}^{-}\right)=0 \vee\left(f\left(a_{2}\right)-f\left(b_{2}\right)\right)=0 \vee\left(-f\left(a_{1}\right)+y-y+f\left(b_{1}\right)\right)=$ $0 \vee\left(-f\left(a_{1}\right)+f\left(b_{1}\right)\right)=f\left(a_{1}^{\sim} \odot b_{1}\right)$. This implies $a_{2} \odot b_{2}^{-}=a_{1}^{\sim} \odot b_{1}$.

By symmetry we have

$$
b_{2} \odot a_{2}^{-}=b_{1}^{\sim} \odot a_{1}=c_{12}=\left(b_{1} \wedge a_{1}\right)^{\sim} \odot a_{1}=\tilde{c_{11}} \odot a_{1} .
$$

Then $c_{12} \wedge c_{21}=\tilde{c_{11}} \odot b_{1} \wedge \tilde{c_{11}} \odot a_{1}=\tilde{c_{11}} \odot\left(b_{1} \wedge a_{1}\right)=0$. Hence $c_{12}+c_{21}=c_{21}+c_{12}$, so that $f\left(a_{1}\right)+f\left(a_{2}\right)=f\left(c_{11}\right)+f\left(c_{12}\right)+f\left(c_{21}\right)+f\left(c_{22}\right)=f\left(c_{11}\right)+f\left(c_{21}\right)+$ $f\left(c_{12}\right)+f\left(c_{22}\right)=f\left(b_{1}\right)+f\left(b_{2}\right)$.

Uniqueness. Adding the elements $f\left(c_{11}\right)$ and $f\left(c_{21}\right)$, respectively, to the equality $f\left(c_{12}\right) \wedge f\left(c_{21}\right)=0$, we obtain $\left(f\left(c_{11}\right)+f\left(c_{12}\right)\right) \wedge\left(f\left(c_{11}\right)+f\left(c_{21}\right)\right)=f\left(c_{11}\right)$, so that $c_{11}=a_{1} \wedge b_{1}$, and similarly $c_{22}=a_{2} \wedge b_{2}$. Using the cancellation property, we see that $c_{12}$ and $c_{21}$ are defined consequently in the same way as at the beginning of the present proof.

The last proposition can be extended as follows.

PROPOSITION 5.2. Let $f\left(a_{1}\right)+\cdots+f\left(a_{m}\right)=f\left(b_{1}\right)+\cdots+f\left(b_{n}\right)$, where $a_{1}, \ldots, a_{m}$ and $b_{1}, \ldots, b_{n}$ are elements of $M$. Then there exist elements $c_{i j}$ in $M$ for $1 \leq i \leq m$ and $1 \leq j \leq n$ such that for all $i$ and $j a_{i}=c_{i 1}+\cdots+c_{i n}$ and $b_{j}=c_{1 j}+\cdots+c_{m j}$. Moreover, we may assume that

$$
\left(c_{i+1, j}+\cdots+c_{m j}\right) \wedge\left(c_{i, j+1}+\cdots+c_{i n}\right)=0
$$

for all $i<m$ and all $j<n$, and under this condition the $c_{i j}$ are uniquely determined.

PROOF. Using the classical result on the Riesz decomposition property [10, page 68] holding in $\ell$-groups, we can find unique elements $c_{i j}^{\prime} \in G^{+}$such that $f\left(a_{i}\right)=$ $c_{i 1}^{\prime}+\cdots+c_{i n}^{\prime}$ and $f\left(b_{j}\right)=c_{1 j}^{\prime}+\cdots+c_{m j}^{\prime}$ which fulfill (5.1). Since $f\left(a_{i}\right) \geq c_{i j}^{\prime}$ for any $i, j$, we conclude from the fact $f(M)=\Gamma\left(G^{\prime}, f(1)\right)$ that there exists a unique element $c_{i j} \in M$ (for all $i$ and all $j$ ) such that $c_{i j}^{\prime}=f\left(c_{i j}\right)$. Since $f$ preserves $\wedge$, we have the assertions in question.

THEOREM 5.3. Let $\left(M ; \oplus, \odot,^{-}, \sim, 0,1\right)$ be a pseudo $M V$-algebra, and $f$ and $G$ those from Theorem 3.9. Then $(G, f)$ is a universal group for $M$.

PROOF. Assume that $h$ is any order and + preserving mapping from $M$ into a group $K$. We define a mapping $\phi: f(M) \rightarrow K$ by $\phi(f(a))=h(a), a \in M$. If $f(a)=f(b)$, then $a=b$ which entails that $\phi$ is a well defined mapping. In addition, if $a=a_{1}+\cdots+a_{n}$, then $\phi(f(a))=\phi\left(f\left(a_{1}\right)\right)+\cdots+\phi\left(f\left(a_{n}\right)\right)$. We extend $\phi$ to a mapping $\hat{\phi}: G^{+} \rightarrow K$ by

$$
\hat{\phi}\left(f\left(a_{1}\right)+\cdots+f\left(a_{m}\right)\right)=\phi\left(f\left(a_{1}\right)\right)+\cdots+\phi\left(f\left(a_{m}\right)\right)
$$


for all $a_{1}, \ldots, a_{m} \in M$.

We show that $\hat{\phi}$ is a well defined mapping. Suppose thus that

$$
f\left(a_{1}\right)+\cdots+f\left(a_{m}\right)=f\left(b_{1}\right)+\cdots+f\left(b_{n}\right),
$$

where $b_{1}, \ldots, b_{n} \in M$. The proof will consists of three steps

Step 1. Let $f\left(a_{1}\right)+f\left(a_{2}\right)=f\left(b_{1}\right)+f\left(b_{2}\right)$. By Proposition 5.1, there exist four elements $c_{11}, c_{12}, c_{21}, c_{22} \in M$ with $c_{12} \wedge c_{21}=0$ such that $f\left(a_{1}\right)=f\left(c_{11}\right)+f\left(c_{12}\right)$, $f\left(b_{1}\right)=f\left(c_{11}\right)+f\left(c_{21}\right), f\left(a_{2}\right)=f\left(c_{21}\right)+f\left(c_{22}\right)$, and $f\left(b_{2}\right)=f\left(c_{12}\right)+f\left(c_{22}\right)$. Then $f\left(c_{12}\right) \wedge f\left(c_{21}\right)=0$, so that $f\left(c_{12}\right)+f\left(c_{21}\right)=f\left(c_{21}\right)+f\left(c_{12}\right)$ and $h\left(c_{12}\right)+h\left(c_{21}\right)=$ $h\left(c_{21}\right)+h\left(c_{21}\right)$. Therefore,

$$
\begin{aligned}
\phi\left(f\left(a_{1}\right)\right)+\phi\left(f\left(a_{2}\right)\right) & =\phi\left(f\left(c_{11}\right)\right)+\phi\left(f\left(c_{12}\right)\right)+\phi\left(f\left(c_{21}\right)\right)+\phi\left(f\left(c_{22}\right)\right) \\
& =\phi\left(f\left(c_{11}\right)\right)+\phi\left(f\left(c_{21}\right)\right)+\phi\left(f\left(c_{12}\right)\right)+\phi\left(f\left(c_{22}\right)\right) \\
& =\phi\left(f\left(b_{1}\right)\right)+\phi\left(f\left(b_{2}\right)\right) .
\end{aligned}
$$

Step 2. Let now (5.2) hold. Choose, by Proposition 5.2, unique elements $c_{i j}$ in $M$, $1 \leq i \leq m, 1 \leq j \leq n$, such that (5.3) hold, that is,

$$
\sum_{i=1}^{m} \sum_{j=1}^{n} f\left(c_{i j}\right)=\sum_{j=1}^{n} \sum_{i=1}^{m} f\left(c_{i j}\right) .
$$

We assert then that

$$
\sum_{i=1}^{m} \sum_{j=1}^{n} \phi\left(f\left(c_{i j}\right)\right)=\sum_{j=1}^{n} \sum_{i=1}^{m} \phi\left(f\left(c_{i j}\right)\right) .
$$

If $n=m=2$, by Step 1, we have (5.4). Suppose thus that the assertion is true for integers $m^{\prime}$ and $n^{\prime}$ with $m^{\prime} \leq m, n^{\prime}<n(n \geq 3)$, and write the equality (5.3) in the form

$$
f\left(a_{1}\right)+\cdots+f\left(a_{m}\right)=f\left(b_{1}\right)+\cdots+f\left(b_{n-2}\right)+\left(f\left(b_{n-1}\right)+f\left(b_{n}\right)\right) .
$$

By the Riesz decomposition property holding in $\ell$-groups, there exist elements $c_{i j}$ in $M(1 \leq i \leq m, 1 \leq j \leq n-2)$ and elements $d_{i}^{\prime} \in G^{+}(1 \leq i \leq m)$ such that

$$
f\left(a_{i}\right)=f\left(c_{i 1}\right)+\cdots+f\left(c_{i, n-2}\right)+d_{i}^{\prime}, \quad f\left(b_{j}\right)=f\left(c_{1 j}\right)+\cdots+f\left(c_{m j}\right)
$$

for $1 \leq j \leq n-2$, and $f\left(b_{n-1}\right)+f\left(b_{n}\right)=d_{1}^{\prime}+\cdots+d_{m}^{\prime}$. Since $d_{i}^{\prime} \leq f\left(a_{i}\right)$, in view of $f(M)=\Gamma\left(G^{\prime}, f(1)\right)$, there exists a unique element $d_{i} \in M$ such that $d_{i}^{\prime}=f\left(d_{i}\right)$, so that (5.3) holds. By the induction hypothesis, we have

$$
\begin{aligned}
& \sum_{i=1}^{m}\left[\phi\left(f\left(c_{i j}\right)\right)+\cdots+\phi\left(f\left(c_{i, n-2}\right)\right)+\phi\left(f\left(d_{i}\right)\right)\right] \\
& \quad=\sum_{j=1}^{n-2}\left[\phi\left(f\left(c_{1 j}\right)\right)+\cdots+\phi\left(f\left(c_{m j}\right)\right)\right]+\left[\phi\left(f\left(d_{1}\right)\right)+\cdots+\phi\left(f\left(d_{m}\right)\right)\right] .
\end{aligned}
$$


Applying induction to $f\left(b_{n-1}\right)+f\left(b_{n}\right)=f\left(d_{1}\right)+\cdots+f\left(d_{m}\right)$, there exists elements $c_{i j}$ in $M, 1 \leq i \leq m, j=n-1, n$, with the property $d_{i}=c_{i, n-1}+c_{i n}, b_{j}=c_{1 j}+\cdots+c_{m j}$. The induction hypothesis yields $\left(c_{i+1, j}+\cdots+c_{m j}\right) \wedge\left(c_{i, j+1}+\cdots+c_{i, n-2}+d_{i}\right)=0$ for $i<m$ and $j \leq n-2$ and $\left(c_{i+1, n-1}+\cdots+c_{m, n-1}\right) \wedge c_{i n}=0$, respectively. Hence

$$
\phi\left(f\left(d_{1}\right)\right)+\cdots+\phi\left(f\left(d_{m}\right)\right)=\sum_{i=1}^{m} \phi\left(f\left(c_{i, n-1}\right)\right)+\sum_{i=1}^{m} \phi\left(f\left(c_{i n}\right)\right),
$$

which entails that (5.3) and (5.4) hold, consequently $\hat{\phi}$ is a well-defined mapping on $G^{+}$. Moreover, it is additive on $G^{+}$.

Step 3. We extend now $\hat{\phi}$ to a mapping $\bar{\phi}$ from $G$ into $K$ by $\bar{\phi}(a)=\phi\left(a^{+}\right)-\phi\left(a^{-}\right)$, where $a^{+}:=a \vee 0$ and $a^{-}:=(-a) \vee 0$. Then $\bar{\phi}(-a)=-\bar{\phi}(a)$ for any $a \in G$. We show that $\phi$ is a group homomorphism from $G$ into $K$.

(1) Let $a=a_{1}-a_{2}$ where $a_{1}, a_{2} \in G^{+}$. Then $\bar{\phi}(a)=\phi\left(a_{1}\right)-\phi\left(a_{2}\right)$.

Indeed, we have

$$
\begin{aligned}
a & =a_{1}-a_{2}, & a^{+}-a^{-}=a_{1}-a_{2}, & \left(a^{+} \wedge a^{-}=0\right) \\
-a^{-}+a^{+}= & a_{1}-a_{2}, & a^{+}+a_{2}=a^{-}+a_{1}, & \\
\phi\left(a^{+}\right)+\phi\left(a_{2}\right) & =\phi\left(a^{-}\right)+\phi\left(a_{1}\right), & \phi\left(a^{+}\right)-\phi\left(a^{-}\right) & =\phi\left(a_{1}\right)-\phi\left(a_{2}\right), \\
-\phi\left(a^{-}\right)+\phi\left(a^{+}\right) & =\phi\left(a_{1}\right)-\phi\left(a_{2}\right), & \bar{\phi}(a) & =\phi\left(a_{1}\right)-\phi\left(a_{2}\right) .
\end{aligned}
$$

(2) If $a=-b_{1}+b_{2}$, where $b_{1}, b_{2} \in G^{+}$, then $\bar{\phi}(a)=-\phi\left(b_{1}\right)+\phi\left(b_{2}\right)$.

Indeed $a^{+}-a^{-}=-b_{1}+b_{2}$, then $a^{+}=-b_{1}+b_{2}+a^{-}$and $b_{1}+a^{+}=b_{2}+a^{-}$. Hence, $\phi\left(b_{1}\right)+\phi\left(a^{+}\right)=\phi\left(b_{2}\right)+\phi\left(a^{-}\right)$and $\bar{\phi}(a)=\phi\left(a^{+}\right)-\phi\left(a^{-}\right)=-\phi\left(b_{1}\right)+\phi\left(b_{2}\right)$.

(3) If $a \in G$ and $b \in G^{+}$, then $\bar{\phi}(a+b)=\bar{\phi}(a)+\phi(b)$.

Indeed, $\bar{\phi}(a+b)=\bar{\phi}\left(a^{+}-a^{-}+b\right)=\bar{\phi}\left(-a^{-}+\left(a^{+}+b\right)\right)$ and by $(2), \bar{\phi}(a+b)=$ $-\phi\left(a^{-}\right)+\left(\phi\left(a^{+}\right)+\phi(b)\right)=\bar{\phi}(a)+\phi(b)$.

(4) $\bar{\phi}(a-b)=\bar{\phi}(a)-\phi(b)$ if $a \in G, b \in G^{+}$.

Calculate, $\bar{\phi}(a-b)=\bar{\phi}\left(a^{+}-a^{-}-b\right)=\bar{\phi}\left(a^{+}-\left(b+a^{-}\right)\right)$and by (3), $\bar{\phi}(a-b)=$ $\phi\left(a^{+}\right)-\left(\phi(b)+\phi\left(a^{-}\right)\right)=\bar{\phi}(a)-\phi(b)$.

(5) Let $a, b \in G$. Then $\bar{\phi}(a+b)=\bar{\phi}\left(\left(a+b^{+}\right)-b^{-}\right)=\bar{\phi}\left(a+b^{+}\right)-\phi\left(b^{-}\right)=$ $\bar{\phi}(a)+\phi\left(b^{+}\right)-\phi\left(b^{-}\right)=\bar{\phi}(a)+\bar{\phi}(b)$, which finally proves that $(G, f)$ is a universal group for $M$.

THEOREM 5.4. Let $u$ be a strong element of an $\ell$-group $G$. Then $(G, i d)$ is a universal group for the pseudo $M V$-algebra $\Gamma(G, u)$, where id is the natural embedding of $\Gamma(G, u)$ into $G$.

PROOF. It is clear that $i d$ preserves + , and $\Gamma(G, u)$ is generating for $G^{+}$. If now $h$ is any mapping from $\Gamma(G, u)$ into a group $K$ which preserves + , the mapping 
$\phi: \Gamma(G, u) \rightarrow K$ defined by $\phi(a)=h(a)$ can be in analogous way as in the proof of Theorem 5.3 uniquely extended to a group homomorphism $\bar{\phi}$ from $G$ into $K$ such that $h(a)=\bar{\phi}(a), a \in \Gamma(G, u)$.

\section{Categorical equivalence}

In this section, we show that the category of pseudo MV-algebras is categorically equivalent with the category of unital $\ell$-groups.

Let $\mathscr{P} \mathscr{M} \mathscr{V}$ be the category of pseudo MV-algebras whose objects are pseudo MV-algebras $\left(M ; \oplus,^{-}, \sim, 0,1\right)$ and morphisms are pseudo $M V$-homomorphisms, that is, any mapping $f$ from a pseudo $\mathrm{MV}$-algebra $(M ; \oplus,-, \sim, 0,1)$ into $\left(M_{1} ; \oplus_{1},{ }^{-1},{ }^{\sim}\right.$, $\left.0_{1}, 1_{1}\right)$ which preserves $\oplus,-, \sim, 0$ and 1 . Denote by $\mathscr{U} \mathscr{L} \mathscr{G}$ the category of unital $\ell$ groups whose objects are unital $\ell$-groups, that is pairs $(G, u)$ where $u$ is a distinguished strong unit of an $\ell$-group $G$. Its morphisms are unital $\ell$-group homomorphisms, that is, any mapping $f$ from a unital $\ell$-group $(G, u)$ into a unital $\ell$-group $(H, v)$ such that (i) $f(x \pm y)=f(x) \pm f(y)$, (ii) $f(x \vee y)=f(x) \vee f(y)$ and $f(x \wedge y)=f(x) \wedge f(y)$, and (iii) $f(u)=f(v)$.

We define a mapping $\Gamma: \mathscr{U} \mathscr{L} \mathscr{G} \rightarrow \mathscr{P} \mathscr{M} \mathscr{V}$ by: $\Gamma:(G, u) \mapsto \Gamma(G, u)$, for any object $(G, u)$ in $\mathscr{U} \mathscr{L} \mathscr{G}$, where $\Gamma(G, u)$ is defined by $(1.1)$, and if $h$ is a unital $\ell$-homomorphism from $(G, u)$ into $(H, v)$, then $\Gamma(h)(a):=h(a), a \in \Gamma(G, u)$, is a morphism from $\Gamma(G, u)$ into $\Gamma(H, v)$. It is easy to verify that $\Gamma$ is indeed a functor from $\mathscr{U} \mathscr{L} \mathscr{G}$ into $\mathscr{P} \mathscr{M} \mathscr{V}$.

PROPOSITION 6.1. $\Gamma$ is a faithful and full functor from the category $\mathscr{U} \mathscr{L} \mathscr{G}$ into the category $\mathscr{P} \mathscr{M} \mathscr{V}$ of pseudo $M V$-algebras.

PROOF. Let $h_{1}$ and $h_{2}$ be two morphisms from $(G, u)$ into $(H, v)$ such that $\Gamma\left(h_{1}\right)=$ $\Gamma\left(h_{2}\right)$. Then $h_{1}(x)=h_{2}(x)$ for any $x \in \Gamma(G, u)$. Since $\Gamma(G, u)$ generates $G^{+}$, it is clear that $h_{1}(x)=h_{2}(x)$ for any $x \in G$.

To prove that $\Gamma$ is a full functor, suppose that $f$ is a morphism from $\Gamma(G, u)$ into $\Gamma(H, v)$, that is, $f$ is a pseudo MV-homomorphism from $\Gamma(G, u)$ into $\Gamma(H, u)$. Then $f$ preserves the partial addition + and $\wedge$ in $\Gamma(G, u)$, and + coincides with the usual group addition taken in $G$. Since $\Gamma(G, u)$ generates $G$, using the same ideas as in the proof of Theorem 5.3 for constructing the group homomorphism $\bar{\phi}$, we can show that $f$ can be uniquely extended to a group homomorphism $\hat{f}$ from $G$ into $H$.

We claim that $\hat{f}$ is a unital $\ell$-group homomorphism. The proof will proceed in several steps, and it follows mainly ideas of Section 3 .

Step 1. Let $a, b, u_{0} \in G^{+}$. If $\hat{f}(a \wedge b)=\hat{f}(a) \wedge \hat{f}(b)$ and if $\hat{f}\left(u_{0} \wedge(b-(a \wedge b))\right)=$ $\hat{f}\left(u_{0}\right) \wedge \hat{f}(b-(a \wedge b))$, then

$$
\hat{f}\left(\left(a+u_{0}\right) \wedge b\right)=\hat{f}\left(a+u_{0}\right) \wedge \hat{f}(b) .
$$


Indeed, we have

$$
\begin{aligned}
u_{0} \wedge(b-(a \wedge b))+(a \wedge b) & =\left(u_{0}+a \wedge b\right) \wedge b \\
& =\left(u_{0}+a\right) \wedge\left(u_{0}+b\right) \wedge b=\left(u_{0}+a\right) \wedge b,
\end{aligned}
$$

which gives

$$
\begin{aligned}
& \hat{f}\left(\left(a+u_{0}\right) \wedge b\right)=\hat{f}\left(u_{0} \wedge(b-(a \wedge b))\right)+\hat{f}(a \wedge b) \\
& =\left[\hat{f}\left(u_{0}\right) \wedge(\hat{f}(b)-(\hat{f}(a) \wedge \hat{f}(b)))\right]+(\hat{f}(a) \wedge \hat{f}(b)) \\
& \left.=\left[\hat{f}\left(u_{0}\right)+(\hat{f}(a) \wedge \hat{f}(b))\right)\right] \wedge \hat{f}(b) \\
& =\left(\hat{f}\left(u_{0}\right)+\hat{f}(a)\right) \wedge\left(\hat{f}\left(u_{0}\right)+\hat{f}(b)\right) \wedge \hat{f}(b)=\hat{f}\left(a+u_{0}\right) \wedge \hat{f}(b) .
\end{aligned}
$$

Step 2. $\hat{f}(a \wedge b)=\hat{f}(a) \wedge \hat{f}(b)$ whenever $a \in G^{+}$and $b \in \Gamma(G, u)$.

Since $\Gamma(G, u)$ is generating for $G^{+}, a$ is of the form $a=a_{1}+\cdots+a_{n}$ for some $a_{1}, \ldots, a_{n} \in \Gamma(G, u)$. We will use the mathematical induction on $n$.

If $n=1$, the statement is trivial. Suppose now that the statement holds for any $a^{\prime}=a_{1}+\cdots+a_{i}$ with $1 \leq i \leq n$. Put $a=a_{1}+\cdots+a_{n}, u_{0}=a_{n+1}$. Then there exist $v_{1}, \ldots, v_{k} \in \Gamma(G, u)$ such that $b=\left(v_{1}+\cdots+v_{k}\right)+(a \wedge b)$. Since $v:=v_{1}+\cdots+v_{k} \leq b \in \Gamma(G, u), v \in \Gamma(G, u)$. Hence $v=b-(a \wedge b)$. Since $\hat{f}$ preserves meets in $\Gamma(G, u)$, we have $\hat{f}\left(u_{0} \wedge v\right)=\hat{f}\left(u_{0}\right) \wedge \hat{f}(v)$, so that $\hat{f}\left(u_{0} \wedge(b-(a \wedge b))\right)=\hat{f}\left(u_{0}\right) \wedge \hat{f}(b-(a \wedge b))=\hat{f}\left(u_{0}\right) \wedge(\hat{f}(b)-(\hat{f}(a) \wedge \hat{f}(b)))$, where we have used induction hypothesis for $a$ and $b$. By Step $1, \hat{f}\left(\left(a+u_{0}\right) \wedge b\right)=$ $\hat{f}\left(a+u_{0}\right) \wedge \hat{f}(b)$, that is, $\hat{f}\left(\left(a_{1}+\cdots+a_{n+1}\right) \wedge b\right)=\hat{f}\left(a_{1}+\cdots+a_{n+1}\right) \wedge \hat{f}(b)$ for any $n$.

Step 3. $\hat{f}(a \wedge b)=\hat{f}(a) \wedge \hat{f}(b)$ whenever $a, b \in G^{+}$.

Let $a=a_{1}+\cdots+a_{n}, b=b_{1}+\cdots+b_{k}$. The proof will follow the complete induction on $k$.

If $k=1$, we apply Step 2. Suppose now that the assertion holds for any $j$ with $1 \leq j \leq k$. Put $B=a, A=b_{1}+\cdots+b_{k}, u_{0}=b_{k+1}$. By Step 2, $\hat{f}\left(u_{0} \wedge(B-\right.$ $(A \wedge B)))=\hat{f}\left(u_{0}\right) \wedge \hat{f}(B-(A \wedge B))$ and $\hat{f}(A \wedge B)=\hat{f}(A) \wedge \hat{f}(B)$. Therefore the conditions of Step 1 are satisfied, so that $\hat{f}\left(\left(A+u_{0}\right) \wedge B\right)=\hat{f}\left(A+u_{0}\right) \wedge \hat{f}(B)$ which proves $\hat{f}\left(\left(a_{1}+\cdots+a_{n}\right) \wedge\left(b_{1}+\cdots+b_{k+1}\right)\right)=\hat{f}\left(a_{1}+\cdots+a_{n}\right) \wedge \hat{f}\left(b_{1}+\cdots+b_{k+1}\right)$ for each $n$ and each $k$.

Step 4. $\hat{f}(a \wedge b)=\hat{f}(a) \wedge \hat{f}(b)$ whenever $a, b \in G$. Then $a=a^{+}-a^{-}$and $b=b^{+}-b^{-}$, and $a=-a^{-}+a^{+}, b=-b^{-}+b^{+}$. By Step 3, $\hat{f}\left(\left(a^{+}+b^{-}\right) \wedge\left(a^{-}+b^{+}\right)\right)=$ $\hat{f}\left(a^{+}+b^{-}\right) \wedge \hat{f}\left(a^{-}+b^{+}\right)$. Subtracting $\hat{f}\left(b^{-}\right)$from the right-hand side and $\hat{f}\left(a^{-}\right)$ from the left-hand side, we obtain the assertion in question.

PROPOSITION 6.2. The functor $\Gamma$ from the category $\mathscr{U} \mathscr{L} \mathscr{G}$ into the category $\mathscr{P} \mathscr{M} \mathscr{V}$ is right-adjoint. 
Proof. We show that given a pseudo MV-algebra $\left(M ; \oplus,^{-}, \sim, 0,1\right)$ there is a universal arrow $((G, u), f)$. That is, $f$ is a pseudo MV-homomorphism from $M$ into $\Gamma(G, u)$ such that if $(H, v)$ is an object from $\mathscr{U} \mathscr{L} \mathscr{G}$ and of $h$ is a pseudo MVhomomorphism from $M$ into $\Gamma(H, v)$, then there exists a unique morphism $f^{*}$ from $(G, u)$ into $(H, v)$ such that $\Gamma\left(f^{*}\right) \circ f=h$.

Due to Theorem 5.3, there is a universal group $(G, f)$ for $M$ such that $f$ : $M \rightarrow \Gamma(G, u)$ is a pseudo MV-isomorphism. In addition, if $h$ is a pseudo MVhomomorphism from $M$ into $\Gamma(H, v)$, then $h$ is also a mapping from $M$ into $H$ preserving + in $M$. Due to Theorem 5.3, there is a unique group homomorphism $\phi$ from $G$ into $H$ such that $\phi(f(a))=h(a), a \in M$. Using ideas of the proof of Proposition 6.1, $\phi$ preserves also $\wedge$ in $G$ so that $f^{*}=\phi$ is a unital $\ell$-group homomorphism in question.

Now we define the converting functor $\mathscr{G}$ from $\mathscr{P} \mathscr{M} \mathscr{V}$ into $\mathscr{U} \mathscr{G}$ by $\Gamma(M):=$ $(G(M), u)$, where $(G(M), u)$ is that from Theorem 3.9, and if $f$ is a pseudo MVhomomorphism from $M$ into $M_{1}$, then $\mathscr{G}(h)$ is a unique $\ell$-group homomorphism derived in the proof of Proposition 6.2. Therefore, we conclude the following statement.

PROPOSITION 6.3. $\mathscr{G}$ is a functor from the category $\mathscr{P} \mathscr{M} \mathscr{V}$ into the category $\mathscr{U} \mathscr{L} \mathscr{G}$ which is left-adjoint.

We can now prove the main statement of the present section.

THEOREM 6.4. $\Gamma$ is a categorical equivalence of the category $\mathscr{U} \mathscr{L} \mathscr{G}$ of unital $\ell$-groups and of the category $\mathscr{P} \mathscr{M} \mathscr{V}$ of pseudo $M V$-algebras.

PROOF. According to [13, Theorem IV.4.1 (i), (iii)], it is necessary to show that for any pseudo $\mathrm{MV}$-algebra $(M ; \oplus,-, \sim, 0,1)$ there is an object $(G, u)$ in $\mathscr{U} \mathscr{L} \mathscr{G}$ such that $\Gamma(G, u)$ is isomorphic to $M$. To show that it is sufficient to take any universal group $(G, f)$ of $M$, and take the object $(G, u)$, where $u=f(1)$. By Theorem 3.9, $u$ is a strong unit, and by Theorem 5.3, $h$ is an isomorphism in question.

As a corollary we have the crucial result of Mundici $[14,5]$ saying that the category of Abelian unital $\ell$-groups $\mathscr{A} \mathscr{U} \mathscr{L} \mathscr{G}$ is categorically equivalent with the category $\mathscr{M} \mathscr{V}$ of MV-algebras which is here proved by a way different of Mundici's one.

COROLlaRY 6.5. The restriction of $\Gamma$ on $\mathscr{A} \mathscr{U} \mathscr{L} \mathscr{G}$ defines a categorical equivalence of Abelian unital $\ell$-groups and the category $\mathscr{M} \mathscr{V}$ of $M V$-algebras. 


\section{Acknowledgement}

The author is indebted to Professors J. Jakubík, D. Mundici and A. Iorgulescu for the fruitful discussion on the subject, and the referees for their valuable suggestions which improved the readability of the paper.

This research was supported by the grant VEGA No 2/7193/20 of the Slovak Academy of Sciences, Slovakia.

\section{References}

[1] R. Baer, 'Free sums of groups and their generalizations. An analysis of the associative law', Amer. J. Math. 41 (1949), 706-742.

[2] G. Birkhoff, Lattice theory, Amer. Math. Soc. Coll. Publ. 25 (Amer. Math. Soc., Providence, RI, 1997).

[3] B. Bosbach, 'Concerning semiclans', Arch. Math. 37 (1981), 316-324.

[4] C. C. Chang, 'Algebraic analysis of many-valued logics', Trans. Amer. Math. Soc. 88 (1958), $467-490$.

[5] R. Cignoli, I. M. L. D'Ottaviano and D. Mundici, Algebraic foundations of many-valued reasoning (Kluwer, Dordrecht, 2000).

[6] M. R. Darnel, Theory of lattice-ordered groups (Marcel Dekker, New York, 1995).

[7] A. Dvurečenskij, 'On partial addition in pseudo MV-algebras', in: Proc. Fourth Inter. Symp. on Econ. Inform., May 6-9, 1999, Bucharest (ed. I. Smeureanu et al.) (INFOREC Printing House, Bucharest, 1999) pp. 952-960.

[8] A. Dvurečenskij and M. G. Graziano, 'Remarks on representations of minimal clans', Tatra Mt. Math. Publ. 15 (1998), 31-53.

[9] A. Dvurečenskij and S. Pulmannová, New trends in quantum structures (Kluwer, Dordrecht and Ister Science, Bratislava, 2000).

[10] L. Fuchs, Partially ordered algebraic systems (Pergamon Press, Oxford, 1963).

[11] G. Georgescu and A. Iorgulescu, 'Pseudo MV algebras', Mult.-Valued Log. 6 (2001), 95-135.

[12] U. Höhle, 'Commutative, residuated l-monoids', in: Non-classical logic and their representation to fuzzy subsets (eds. U. Höhle and E. P. Klement) (Kluwer, Dordrecht, 1995) pp. 53-106.

[13] S. MacLane, Categories for the working mathematician (Springer, New York, 1971).

[14] D. Mundici, 'Interpretation of AF $C^{*}$-algebras in Łukasiewicz sentential calculus', J. Funct. Anal. 65 (1986), 15-63.

[15] J. Rachůnek, 'A non-commutative generalization of MV-algebras', Czechoslovak Math. J., to appear.

[16] J. Schmidt, 'Zur Kennzeichnung der Dedekind-MacNeilleschen Hülle einer geordneten Menge', Arch. Math. 7 (1956), 241-249.

[17] O. Wyler, 'Clans', Compos. Math. 17 (1966/67), 172-189.

Mathematical Institute

Slovak Academy of Sciences

Štefánikova 49

SK-814 73 Bratislava

Slovakia

e-mail: dvurecen@mat.savba.sk 
